\title{
Hacia un nuevo paradigma de la comunicación en las organizaciones
}

\author{
Jesús Tovar MendozA*
}

\section{Introducción}

El presente ensayo desarrollará una síntesis de un marco teórico de las organizaciones basado en un enfoque socio-lingüístico, es decir una nueva mirada de las organizaciones como redes recurrentes de compromisos humanos que pasan por el lenguaje.

El lenguaje es más de lo que dicen las palabras, el lenguaje es una fuerza activa que además de reflejar el mundo que le antecede, reproduce y constituye nuevos mundos. Estos mundos, creados por las palabras, son esencialmente de naturaleza social; en tanto que se trata de acciones que comprometen a individuos y que los obligan entre sí a establecer un marco de interrelaciones de cierta permanencia en el tiempo.

La mayor permanencia de tiempo de estas interrelaciones de actores constituidas por el lenguaje hace posible la configuración de patrones, es decir de acciones previsibles y por lo tanto permiten la existencia de un proceso de estructuración de la acción social. Una de las formas en que esta estructuración se hace posible y visible es a través de las organizaciones, es decir acciones suscitadas por un lenguaje específico y delimitado por fronteras, dentro de las cuales se constituyen coaliciones de intereses que colaboran competitivamente para alcanzar beneficios.

La presentación de este enfoque linguiístico de las organizaciones se basa en las siguientes corrientes teóricas. Por un lado tenemos el aporte de los enfoques que se desprenden del "giro lingüístico" del 2do Wittgenstein y que se plasma con mayor precisión en los trabajos de John Austin y John Searle. De manera estrechamente relacionada se recogen construcciones filosóficas de Nietszche y Heidegger. La agrupación de estas ideas en un conjunto referido a las organizaciones se debe a los trabajos de teóricos latinoamericanos como Fernando Flores, Rafael Echevarría y Humberto Maturana, a los cuales haremos referencia constante en esta versión sintética de sus propuestas analíticas y normativas, aunque no necesariamente aprobada por los mismos, lo cual implica apreciaciones de índole personal.

* Universidad Intercontinental 


\section{El Giro Lingüístico: Hacia una Ontología del Lenguaje}

La metafísica de origen griego se caracateriza por ser un lenguaje de las ideas, es decir una separación del lenguaje respecto de la acción, y por tanto de la teoría y la práctica. Es una búsqueda permanente de "lo verdadero" a través de la razón, una verdad que es inmutable, no contingente, que no se arraiga en la vida sino en el pensamiento. Este acercamiento a un conocimiento holístico hace que el pensamiento sea la base para entender a los seres humanos, el pensamiento nos convierte en el tipo de ser que somos: "pienso, luego existo" como señala el máximo exponente de esta metafísica racionalista: Descartes. Así, según esta perspectiva, el pensamiento se convierte en la base del ser, es lo que nos hace humanos.

Las críticas a este planteamiento metáfisico de la filosofia racionalista provienen de tres grandes fuentes, en primer lugar (cronológicamente) está Frederich Nietszche, quien hace un abordaje a la comprension del alma humana desde fuera de los marcos metafísicos. En segundo lugar, la fenomenología existencial de Martín Heidegger que critica los supuestos del cartesianismo, empezando con el más fuerte: la predominancia racional del ser humano. Finalmente, tenemos al "segundo" Wittgenstein que ofrece una comprensión completamente nueva del lenguaje y su rol frente a la realidad.

Éste proceso de crítica es el que luego se denominó el "giro lingüístico", ya que el lenguaje toma el lugar de privilegio, que por siglos había ocupado la razón. Esta evolución del pensamiento se retroalimenta e influye campos como la teoría de sistemas y el paradigma comunicativo en las ciencias sociales, la lingüística y la biología (donde se postula que el rasgo básico de la especie humana es el lenguaje con los aportes de Ernst Mayr y Humberto Maturana).

Una nueva ontología resulta de pensar la naturaleza humana en torno al lenguaje en vez de la centralidad del pensamiento racional. Incluso el propio término "ontología" deviene de su sentido original griego "comprensión del ser en cuanto tal" a una "comprensión de lo que significa el ser humano", lo cual nos remite a una dimensión interpretativa del ser humano, que revela no sólo al sujeto interpretado sino además al sujeto que interpreta. De esta manera cuando decimos algo de alguien, nos colocamos en una situación de compromiso por el cual hemos de sostener y argumentar lo que decimos; así cualquier 
postulado sobre los seres humanos, implica a la vez una comprensión del ser que formula ese postulado.

John Searle (1979) señala que cuando hablamos ejecutamos un número restringido y específico de acciones. Estas acciones las llamó "actos de habla", de esta manera además propuso una taxonomía de estos actos de habla, lo que permitió la posibilidad de "observar" al propio lenguaje y distinguir las diferentes acciones que ejecutamos cuando nos comunicamos. El conocimiento se desplaza así desde lo observado hacia el observador, para conocer a quién habla y cómo se compromete con lo que dice. En síntesis, cada planteamiento hecho por un observador nos dice del tipo de observador que ese observador considera que es.

Asimismo, la orientación interpretativa del ser humano, nos remite a un criterio fenomenológico, según el cual no sabemos lo que las cosas son, sino sólo como las interpretamos u observamos. Vivimos en mundos interpretativos, mundos que cambian constantemente. Por tanto, no es posible encontrar una verdad, ni un propietario de aquella verdad, nos acercamos y nos alejamos de ella con los devenires de nuestras propias actitudes y juicios, que van generando cambios en la realidad y en nuestra forma de ser. Las verdades no resultan fuera del observador, la verdad contingente mora con nosotros mismos, con lo que hacemos y decimos.

La nueva ontología del lenguaje señala que lo que decimos o hacemos revela nuestro juicio sobre nosotros mismos. Tres nuevos postulados complementan esta Ontología:

1) Interpretamos a los seres humanos como seres lingüísticos, es decir son seres que viven en el lenguaje, y por medio de éste se constituyen en cuanto tales. Esto no implica asumir una visión reduccionista que prescinda de cualquier otra dimensión además del lenguaje, de los cuales esta teoría reconoce los que denomina "dominio de las emociones" y "dominio del cuerpo". Pero es a través de lenguaje que podemos tener acceso a fenómenos no lingüísticos, como dice Nietzsche "el lenguaje es una prisión de la cual no podemos escapar". Habitamos en el lenguaje.

2) Interpretamos al Lenguaje como generativo ya que hace que sucedan las cosas; lo cual implica dejar de lado el rol pasivo del lenguaje como reflejo descriptivo de la realidad. El lenguaje crea realidades, es acción, y en el mediano plazo modela nuestra identi- 
dad y el mundo en que vivimos. La identidad personal y social, es sobre todo una construcción lingüística.

3) Los seres humanos se crean así mismos en el lenguaje y a través de él, nadie tiene una forma de ser determinada, y jugamos un rol importante en el diseño y construcción del ser humano que deseamos. El lenguaje nos permiten reinventarnos como identidades sociales.

\section{La Construcción Lingüística de la Realidad}

Un objeto es una relación lingüística que establecemos con el mundo. Los objetos son constituídos en el lenguaje, y fuera del mismo no existe un lugar en el que podamos apoyarnos. Los seres humanos vivimos en mundos lingüísticos de entidades lingüísticas. Es en el lenguaje donde anida cualquier racionalidad, no en la mente como creían los metafísicos. La misma razón es una función del lenguaje.

La existencia del lenguaje implica un dominio consensual para efectuar un tipo particular de comunicación, que permite a su vez que miembros de una especie coordinen acciones comunes. Pero el lenguaje es un tipo especial de coordinación, es la coordinación de la coordinación del comportamiento, es decir cuando los miembros participantes de una acción coordinan la forma en que van a coordinar juntos la acción siguiente, es en síntesis una coordinación recursiva del comportamiento.

A diferencia de los animales que también poseen sistemas de lenguajes, los seres humanos tienen un número muy grande de signos consensuales, pero sobre todo, por que tenemos la capacidad para "hacer girar el lenguaje sobre sí mismo". Ello implica que podemos hablar sobre lo que hablamos, sobre la forma en que coordinamos nuestra coordinación, una y otra vez. Es esta recursividad del lenguaje lo que nos permite observarnos a nosotros mismos como observadores.

A partir de una pregunta simple a cualquier persona en un encuentro social: ¿quién eres?, nos encontramos con un relato. Nuestra identidad esta asociada a la forma en que manifestamos el relato de nosotros mismos. Si modificamos algún aspecto de un relato anterior, estamos modificando nuestra identidad frente a los demás. Como individuos resultamos ser construcciones lingüísticas, relatos que nosotros elaboramos y difundimos mediante el lenguaje. Somos seres racionales por que somos seres lingüísticos viviendo en un mundo lingüístico. 
Pero además, en tanto individuos, somos lo que somos debido a una cultura lingüística en la que crecemos; y a una determinada posición que ocupamos en el sistema general de coordinación al que pertenecemos. Por lo tanto, somos además de construcciones lingüísticas, un fenómeno social; en la medida que las historias que contamos de nosotros están fabricadas de historias generadas históricamente por una comunidad. De esta manera, los seres humanos hablamos y actuamos de acuerdo a los sistemas sociales a los que pertenecemos; sin embargo estos sistemas sociales son continuamente transformados por las acciones de los individuos.

\section{Los actos lingüísticos básicos}

Como ya se señaló anteriomente, al lenguaje en la concepción tradicional le correspondía esencialmente reflejar la realidad: describirla. Y actualmente es nuestro propio sentido común el que da por sentado que el lenguaje describe el estado de las cosas: "Estamos a 50 grados Farenheit", "el bus azul pasa a las 4:30 por el paradero de mi casa", estas frases son pasivas en tanto primero "sucede algo" que luego el lenguaje ha de dar cuenta. El lenguaje parece siempre llegar tarde, cuando la realidad ya se ha instalado. Este tipo de actos de habla se han denominado AFIRMACIONES, es decir son proposiciones de nuestras observaciones.

Las AFIRMACIONES se enfrentan al reto de adecuarse al mundo lo más precisamente posible, por lo tanto es el mundo el que conduce a la palabra. No siempre estas afirmaciones son descripciones que logran ser reflejo perfecto de la realidad, son mas que todo proposiciones acerca de nuestras observaciones. Pero las observaciones son posibles si poseemos distinciones. Sin la distinción "silla" no puedo hacer la observación de una silla, así por ejemplo los esquimales pueden distinguir más distinciones del color blanco de lo que podemos nosotros; por lo que la pregunta ¿cuántos tonos de blanco realmente existe? solo tiene sentido en el marco de una determinada tradición de distinciones.

Más allá de estas diferencias de distinciones entre comunidades que nos remiten a tradiciones sociales e históricas, poseemos los seres humanos una capacidad de compartir lo que observamos, suponemos que nuestras observaciones son la forma como son realmente las cosas. Este compartir nos permite clasificar las afirmaciones entre VERDADERAS o FALSAS, siempre al interior de un "espacio común 
de distinciones", es decir no existe una Verdad, sino que es una distinción colectiva a partir de una convención social que hace posible la coexistencia de la comunidad.

Una afirmación verdadera es una proposición para la cual podemos proporcionar un testigo, el cual ha compartido mi observación. Por tanto, mi observación es falsa en el sentido de que otro sujeto que haya compartido la observación no acuerde con lo que afirmo, resulta finalmente una afirmación sujeta a confirmación por un tercero. No todas las afirmaciones son verdaderas o falsas, algunas veces no se pueden confirmar por no existir las condiciones necesarias para su corroboración, así por ejemplo el siguiente pronóstico de tiempo: "va a llover mañana", requiere que esperemos el momento aludido para confirmar la verdad o falsedad de la afirmación.

Cada vez que ejecutamos un acto de habla adquirimos un compromiso y debemos aceptar la responsabilidad social de lo que decimos. Lo cual hace que el hablar nunca sea inocente. En el caso de las afirmaciones, el compromiso social guarda relación con la necesidad de establecer de manera efectiva que la palabra cumple con la exigencia de adecuarse a las observaciones que hacemos sobre el estado del mundo al que se alude. Este compromiso por la veracidad de las afirmaciones hechas implica tener la posibilidad de proporcionar un testigo que corrobore nuestras observaciones.

Las afirmaciones que nos dan cuenta del mundo nos señalan la capacidad de configurar este mundo por medio del lenguaje. Una persona con una capacidad reducida de lenguaje implicará que tiene a su vez un mundo reducido. El concepto de mundo no resulta geográfico, resulta ser producto de nuestra capacidad de hacer distinciones sobre el acontecer.

Otro tipo muy diferente de actos de habla es lo que conocemos como DECLARACIONES. Cuando hacemos declaraciones no hablamos acerca del mundo, generamos un nuevo mundo para nosotros. La palabra genera un realidad diferente, ya que luego de haber expresado algo, el mundo ya no es el mismo de antes gracias a cierto poder del lenguaje. Un ejemplo de ello es la siguiente declaración: "cuando en el curso de los acontecimientos humanos, llega a ser necesario para un pueblo el disolver los vínculos políticos que lo conectarán con otro", que forma parte de la Declaración de Independencia de los Estados Unidos y que implica no una descripción de una situación, sino la creación de un nuevo mundo. Otro tipo de 
declaraciones son las que pronuncia el juez cuando anuncia su veredicto de "inocente" o cuando el árbitro decreta "penal" o cuando un funcionario anuncia "los declaro marido y mujer". En todos estos casos, el mundo es diferente después de estas declaraciones.

Las declaraciones no están relacionadas con nuestras capacidades compartidas de observación, como acontecía con las afirmaciones; están relacionadas con el poder, es decir que podemos hacer declaraciones si tenemos la capacidad para hacerlas cumplir, ya sea por la fuerza o por habernos sido otorgada alguna autoridad. La autoridad como expresión del poder es asignada por alguna comunidad a ciertas personas para evitar el riesgo de la anarquía y la desintegración.

Las declaraciones no son verdaderas o falsas como en el caso de las afirmaciones. Son válidas o inválidas según el poder de la persona que las hace. Asi, el compromiso que se adquiere cuando se hace una declaración es demostrar la autoridad respectiva del que formula la declaración, y además a comportarnos consistentemente con la nueva realidad que hemos declarado. Sin tal autoridad, las declaraciones no tienen validez, ni por tanto eficacia en su capacidad para transformar la realidad.

Sin embargo, existen ciertas declaraciones que todos podemos hacer y que están asociadas al dominio de nuestra propia individualidad. Es decir, el individuo por el hecho de ser tal puede efectuar declaraciones respecto del dominio de su individualidad. Una sociedad democrática es aquella que respeta, protege y promueve los derechos relacionados con estos dominios de la individualidad.

Algunas de estas declaraciones del ámbito de la autoridad personal son:

La declaración del NO: Declaración que asienta la legitimidad de la persona, ya que tenemos el derecho de no aceptar el estado de cosas que enfrentamos y de las demandas de otros. Forma parte de nuestro inalienable derecho de escoger, elecciones que colectivamente hacen posible la existencia del mercado y la democracia.

La declaración del SI: Declaración que suele operar por "default", ya que mientras no decimos NO, se asume que afirmamos. Sin embargo, la aceptación (implícita o explícita) compromete nuestra credibilidad frente a los demás, ya que pocas cosas afectan la identidad de una persona que aceptar con un SI y no actuar coherentemente con tal declaración. 
La declaración de GRATITUD: El poder generativo de esta declaración, que alude a la satisfacción de alguien que nos ha favorecido o ha cumplido su compromiso con nosotros, facilita el establecimiento de una relación social. Pero no sólo con las personas, ya que agradecer a la vida misma permite la construcción de la identidad, otorgar sentido a nuestra existencia.

La declaración del PERDÓN: Viene a ser el reverso de la gratitud, el perdón implica asumir las responsabilidades de los compromisos que no cumplimos o de los daños causados a otras personas. El perdón no exime de otras acciones de reparación de las consecuencias ocasionadas, por lo contrario abre ese marco de nuevas acciones reconstructivas.

Las declaraciones, a diferencia de las afirmaciones, representan el acto lingüístico por excelencia, es decir el que crea las condiciones para la emergencia de los demás, en la medida que para hacer afirmaciones requerimos de un espacio de distinciones, las cuales se crean por medio de declaraciones. Por ejemplo, afirmar "hoy es miércoles" implica que una comunidad comparta la forma como llamamos los días, para luego poder considerar la verdad o falsedad de tal proposición. Por lo que debió existir un momento en el que por Declaración de alguna autoridad se estableció la convención de llamar a los días de esta determinada manera. Por lo que en rigor, podemos decir que las afirmaciones de nuestras observaciones que operan por medio de distinciones, fueron constituidas por Declaraciones en un momento que podemos denominar "ontogenético".

\section{Figura 1: Relación secuencial del acto lingüístico y la realidad}

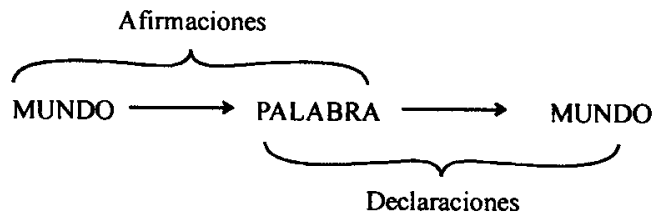

(Fuente: Elaboración propia)

Un tercer tipo de actos lingüísticos son las PROMESAS. Éstas nos permiten coordinar las acciones con otras personas. Cuando alguien hace una promesa se compromete con otra persona a ejecutar alguna acción en el futuro. En es̀a medida, la otra persona puede 
planificar acciones vinculadas con ese anuncio, es decir actúa en el futuro basándose en el hecho de que cuenta con la promesa recibida. Las comunidades, como condición de su coexistencia pacífica, se preocupan de asegurar que las personas cumplan sus promesas, sancionando a los que no lo hacen. Para ello se constituye una extensa legislación sobre contratos, por la cual se refuerza la obligatoriedad de las personas de cumplir con lo estipulado en sus promesas. Nietszche dijo que las personas son animales que hacen promesas.

La efectividad de las promesas incrementa la capacidad de la acción colectiva, se logran resultados que no hubieran sido posibles sin la habilidad de coordinar "mi" acción con la de otros. Por ello se hace necesario comprender cúal es el mecanismo de hacer promesas; el acto de hacer una promesa comprende euatro elementos fundamentales: un orador, un oyente, una acción a llevarse a cabo, un factor tiempo. Una promesa implica dos procesos consecutivos, el acto de hacer la promesa y el acto de cumplirla, el primero es evidentemente comunicativo pero el segundo puede no serlo. El primer proceso implica que las partes involucradas lleguen a un acuerdo sobre lo que se está prometiendo, para lo cual se requiere dos movimientos linguiísticos diferentes: la acción de ofrecer una promesa (oferta) y la acción de aceptarla, o alternativamente la acción de pedir una promesa (petición) y la acción de aceptarla. Si alguien dice: "Prometo ir al cine mañana contigo", esa persona no ha hecho ninguna promesa todavía, hasta que la otra persona no haya dado su consentimiento, que en el caso de no ser positiva, no configura la promesa. La promesa se termina cuando se cierra el proceso de cumplimiento, el cual requiere que la otra persona declare su satisfacción con las condiciones ofrecidas, antes de eso es una promesa pendiente a la espera de su realización.

Cuando hacemos una promesa, nos comprometemos en dos dominios: consistencia y competencia. La consistencia es el juicio que hacemos de que los compromisos públicos contraídos por la persona que hizo la promesa concuerdan con sus compromisos privados. La competencia guarda relación con el juicio de que la persona que hizo la promesa está en condiciones de ejecutarla efectivamente, de modo de proveer las condiciones de satisfacción acordadas. Cuando falta la consistencia o la competencia, la confianza se ve afectada. Como ocurre con los otros actos lingüísticos, siempre somos responsables implícitos de los compromisos sociales de lo que decimos: ya sea que 
afirmemos (veracidad), declaremos (validez) o prometamos (consistencia y competencia).

Finalmente, tenemos un cuarto tipo de acto lingüístico que se denomina JUICIO. Comparemos dos actos lingüisticos: "María tiene 26 años" y "María es bonita", el parecido de ambas asevereciones es que ambas atribuyen propiedades a María, pero el tipo de propiedades atribuidas es diferente, en el primer caso se remite a lo que llamamos un "hecho", mientras que en el segundo implica un "juicio de valor", es decir una opinión, donde no cabe esperar el mismo grado de concordancia con otros interlocutores como si se espera de la descripción de un hecho. Sin embargo, por muchos siglos se han tratado ambas proposiciones de manera similar, de tal forma que tenemos aseveraciones de carácter ético o estético con pretensiones de objetividad, con independencia de los sujetos que formulaban dichas proposiciones.

Ya en este siglo Bertrand Russell señaló que la mayoría de los problemas filosóficos tienen su raiz en errores lógicos o gramaticales, o de la manera singular a como se refiere Ludwig Wittgenstein respecto de ciertos problemas filosóficos que surgen cuando el "lenguaje se va de vacaciones". Muchos laberintos metafísicos se han creado por no distinguir los juicios de las afirmaciones.

Profundizando en estas diferencias, señalaremos que las afirmaciones operan en un espacio de consenso social, por medio del cual los miembros de una comunidad tienen las mismas distinciones respecto de ciertas materias (el tiempo dividido en años, meses, días, horas y minutos, o la medidas de peso, altura y distancia, entre algunos ejemplos de los muchos existentes), distinciones que sirven como criterio para aceptar algo como verdadero o falso. Sin embargo, algunos de estos consensos cambian con el tiempo, por lo cual una afirmación es verdadera "dentro de", y "para" una determinada sociedad (el peso de la historia).

Mientras que los compromisos para los juicios que formulamos es muy diferente; así cuando decimos: Juan es un eficiente administrador, el día está muy agradable, Carlos maneja mal; no describen algo que ya existe objetivamente, sino que son apreciaciones que "viven en la persona" que la formula, es decir, no nos atan con testigos como las afirmaciones, ya que siempre hay lugar para que otra persona discrepe sin generar una ansiedad por determinar quién tiene la única y exclusiva razón. 
La gente está continuamente emitiendo juicios, aún cuando no se les haya otorgado la autoridad para hacerlos (como en el caso de las declaraciones). Y cuando comunican a otros sus juicios, los que lo escuchan siempre pueden descartarlos, basándose en el hecho de que no han otorgado la autoridad para aceptar esos juicios como válidos, y que ellos tienen a su vez una opinión igualmente válida como diferente.

Los juicios también implican un compromiso social para quien los enuncia, el cual es que los mismos se apoyen en alguna argumentación, es decir que sean juicios "fundados". Por consiguiente, los juicios no son válidos o inválidos como las declaraciones, o verdaderos o falsos como las afirmaciones, sino son "fundados" o "infundados" en función a la actitud que adopta el que los formula respecto del compromiso de explicar por qué dice lo que dice.

Así como las afirmaciones nos remiten a una relación de la palabra con un mundo preconstituído (en un tiempo pasado), y las declaraciones establece una relación con un mundo constituído a partir del enunciado emitido (proyección hacia el futuro), el juicio establece una relación bidireccional con ambos tiempos. Si decimos que "Juan es un excelente empleado", nos estamos probablemente basando en ciertos hechos comprobables del pasado (puntualidad, producción de documentos, inasistencias, etc.), pero a su vez este juicio ayuda a constituir la identidad de "Juan", según la cual podrá ser ascendido, recompensado y respetado. Los juicios son un componente de la identidad no sólo de personas, sino también de organizaciones, naciones, etc, que afectan el porvenir de los enjuiciados. Los juicios operan como una brújula que permiten anticipar algunas consecuencias de nuestras acciones o de las otras personas.

A pesar de haber reconocido que no hay juicios verdaderos, porque siempre involucran la posibilidad de la discrepancia; los juicios juegan un rol importante en la vida de una comunidad, ya que la gente es generadora incesante de juicios, se hacen todo el tiempo y sobre todo lo que se observa. Así, cada vez que enfrentamos algo nuevo, comenzamos a emitir juicios inmediatamente. La razón de esta continuidad holística de los juicios es que todos estamos preocupados por el futuro, el cual genera una incertidumbre que es despejada de algún modo por la orientación que nos brindan los juicios que emiten otras personas y los que se generan a partir de nuestra propia observación. 
Un juicio se funda en cinco condiciones básicas:

a) Siempre emitimos un juicio "por o para algo", es decir el juicio emitido ayuda a "visualizar" un futuro, por el cual se abre o se cierra ciertas posibilidades de acción, que pueden afectar positivamente o negativamente a las personas o instituciones enjuiciadas.

b) Cada vez que emitimos un juicio estamos suponiendo que se coteja con ciertos estándares de comportamiento para juzgar el desempeño de los individuos. Estos estándares provienen de ciertas tradiciones, y por tanto un juicio será más compartido para los que forman parte de una misma historia y contexto. Lo que se considera velocidad de un medio de transporte ha cambiado con el tiempo y la tecnología, y lo mismo se puede decir de los estándares de belleza o bondad, mientras que en el mundo de la moda los estándares cambian en sólo pocos meses o semanas. Ahora bien en la medida que nuestros juicios se basan en estándares, podemos decir que detrás de un juicio hecho por una persona, está la influencia y fuerza de una sociedad o grupo social.

c) Cuando emitimos un juicio, generalmente lo hacemos dentro de ciertos dominios particulares de observación y dominios de acción. Dichos dominios son consensos o convenciones sociales que adoptamos por que consideramos que nos ayudan en la coordinación de nuestras acciones. Dominios tales como "transporte público", "conversaciones amicales", "domingos familiares", "sala de cirugía", etc. Así, podemos decir que un juicio está circunscrito a un específico dominio de observación, no es universal y perenne.

d) Un juicio se funda cuando se proveen afirmaciones en relación a lo que estamos juzgando. Si no somos capaces de apoyarnos en afirmaciones, nuestros juicios serán infundados. No podemos apoyar nuestros juicios, en juicios anteriores y así sucesivamente hacia atrás. Así, si decimos que "Karina es buena estudiante" no podemos apoyarnos en argumentos como "Karina es muy inteligente", "Karina es muy trabajadora", "Karina es bella"; son sólo más juicios a su vez no fundados. Tendríamos que decir que "Karina tiene un promedio de 9,5", "Karina recibió felicitaciones escritas de sus maestros", etc.

e) La cantidad de afirmaciones que brindemos tampoco es garantía última de un juicio bien fundado, ya que el juicio opuesto puede tener más afirmaciones. Por lo que habríamos de considerar la 
posibilidad de un juicio contrario del que formulamos y buscar las afirmaciones que la podrían sustentar, de tal manera que al no encontrar fundamentos suficientes, acentuamos nuestra convicción en el juicio original.

Ya hemos señalado anteriormente que cada vez que decimos algo revelamos de alguna forma quienes somos. Esta capacidad del lenguaje de revelar a su emisor, es particularmente cierto respecto de los juicios. $\mathrm{Si}$ alguien dice por ejemplo que "los negros son peligrosos" se revelará más de quién emite el juicio que respecto de los propios negros.

Uno de los dominios a destacar en la emisión de juicios es el de la moral, el cual nos remite al mundo de los valores. Los seres humanos son morales en la medida que buscan permanentemente dar sentido a sus vidas, y una de las maneras es "conferirles valor". Pues bien, es en el terreno de los juicios en el que los seres humanos libran la batalla del sentido de la vida. Es así que Nietszche señala que sin evaluaciones, sin la capacidad de hacer juicios, el núcleo de la existencia estaría vacío (1986). Por lo que los juicios con su capacidad de ofrecernos parámetros de acción para el futuro, representan el núcleo fundamental de la existencia humana. Las afirmaciones con su pretensión de alcanzar la verdad, sólo logran servir como apoyo a los juicios, sobretodo a aquellos que evaluan, y por tanto constituyen, a los seres humanos.

Uno de los temas característicos de la moral refiere a las virtudes y vicios, los cuales descansan por entero en la temática de los juicios. Las virtudes, por ejemplo, son juicios que dentro de una particular comunidad, se seleccionan como los que aseguran la mejor convivencia entre sus miembros, mientras que los vicios contravienen la adecuada convivencia social y por lo tanto se asocian con la degradación de la vida.

En este mismo plano de la moral podemos mencionar cómo a veces los juicios tienen un impacto directo y negativo en la forma de constituir formas de ser, es decir son constituyentes de la identidad de seres individuales y colectivos La primera de ellas se refiere a aquellas personas que se caracterizan por vivir de juicios ajenos, rigiendo su propia existencia a los mismos; eso define lo que llamamos condición de inatenticidad, ya que delegan en los demás la autoridad para emitir los juicios que les importan, nada los alegra más de recibir un juicio positivo, nada los deprime más que obtener una desaprobación. Su vida pasa a depender de fuerzas que no controlan, 
y suele ello traducirse en una condena permanente de incertidumbre. La segunda se refiere a los errores que cometen las personas cuando tratan a los juicios como afirmaciones, obteniendo como resultados: rigidez, intolerancia, conflictos y cierre de oportunidades para el aprendizaje y la innovación. Para ellos los juicios no representan la posibilidad de puntos de vista discrepantes, $y$ un juicio diferente al suyo es tratado como error, relegando a los demas a la esfera de lo equivocado o incluso de "lo malo", es el ambiente típico de los fundamentalismos y la intolerancia. La tercera se refiere a quienes viven sin ser capaces de distinguir entre los juicios fundados y los juicios infundados, de tal manera que ajustan sus vidas a situaciones riesgosas y son sujetos de permanentes decepciones y gran dificultad para diseñar el futuro, ya que se basan en interpretaciones "mágicas" y en optimismos ficticios. Contrariamente a estas percepciones equívocas que generan los juicios, un ser humano libre es aquel que somete sus valores a juicios críticos y fundados, y puede concluir que sus juicios le pertenecen y no que él pertenece a los juicios.

Nuestra forma de emitir expresiones lingüísticas nos remite un juicio particular que influye nuestra forma de convivencia social: la confianza. La cual resulta del juicio que hacemos de los demás o los demás hacen de nosotros a partir de cuan verdaderas son las afirmaciones que hacemos, cuán validas son las declaraciones que realizamos, cuan consistentes y competentes son las promesas que sentamos, cuan fundadas son los juicios que emitimos. En síntesis, el juicio de la confianza se construye a partir de los actos lingüísticos que ejecutamos, y de los compromisos que ellos generan:

Cuadro 1:Clasificación de los Actos Lingüísticos y sus Consecuencias

\begin{tabular}{|c|c|c|c|}
\hline $\begin{array}{l}\text { Tipología de Actos } \\
\text { Lingüisticos }\end{array}$ & $\begin{array}{c}\text { Evaluación del } \\
\text { Acto Lingüístico }\end{array}$ & $\begin{array}{l}\text { Compromiso de } \\
\text { sustentación }\end{array}$ & $\begin{array}{l}\text { Juicio de que Con- } \\
\text { flanza genera al emisor }\end{array}$ \\
\hline Afirmaciones & Verdadero o Falso & Pruebas y Testigos & Veracidad \\
\hline Declaraciones & Válidas o Inválidas & Autoridad & Credibilidad \\
\hline Promesas & $\begin{array}{l}\text { Sincera o Insincera } \\
\text { Posible o Imposible }\end{array}$ & $\begin{array}{c}\text { Consistencia } \\
y \\
\text { Competencia }\end{array}$ & Confiabilidad \\
\hline Juicios & Fundado o Infundado & Argumentos & Solidez \\
\hline
\end{tabular}

(Fuente: Elaboración propia).

Nuestra impecabilidad en el respeto a los compromisos involucrados en cada acto lingüístico es la base que nos permite 
construir la confianza que los demás tengan en nosotros y viceversa en la que nosotros tengamos en los demás. Por tanto, la confianza resulta ser también un fenómeno lingüístico, por el cual aceptamos a los demás como seres legítimamente diferentes y autónomos en sus capacidades de actuar, una confianza que no sólo se traduce en actos de habla sino en toda nuestra forma de comportamiento en que estamos en relación con otros. Lo cual nos lleva finalmente, a un tema fundamental en la ontología del lenguaje: la ontología del lenguaje se sustenta en una determinada ética de la convivencia basada en la confianza mutua.

\section{Hacia una teoría linguística de la acción}

Los supuestos metafísicos racionales sobre la acción humana son: aquel que sostiene que todo sujeto se halla expuesto a la presencia e inmediatez física del mundo de los objetos (res extensa) y aquel que sóstiene que define al ser humano como un ser eminentemente racional en su actuar del mundo (res cogitans). Esta concepción hace de la relación sujeto-objeto el principal problema a dilucidar en la filosofía moderna, es decir determinar la posibilidad de que el sujeto pueda alcanzar una fiel representación del mundo de los objetos, o sea el tema del conocimiento (gnoseología).

Esta perspectiva nos conduce a una comprensión racionalista de la acción humana, ya que asumimos la razón o el pensamiento como anteriores a la acción, es decir actuamos en conciencia, por tanto toda acción humana es acción racional, así podemos explicar la acción a través de encontrar la razón que la motivó. Aún en aquellas acciones aparentemente irracionales (por ejemplo los actos fallidos) que se ejecutan sin deliberación explícita previa, son abordadas a partir de una exploración de razones inconscientes, de tal manera que sigue prevaleciendo el postulado de que la razón conduce a la acción.

Desde la perspectiva ontológica lingüística, la razón es un tipo de experiencia humana derivada del lenguaje. Incluso la ciencia y la filosofía se asumen novedosamente como actividades interpretativas efectuadas por los individuos desde dentro del lenguaje. Así, la razón pertenece al dominio de nuestras explicaciones, no pertenece al fenónemo en sí, son los seres humanos los que forjan una explicación que procura dar cuenta del fenómeno. La razón siempre guarda relación con el observador y no con lo observado. Frente al principio de Leibniz "todo tiene una razón", se replica: los seres humanos 
pueden a través del lenguaje proveer de razones para todo lo que acontece.

Martín Heidegger $(1982,1985)$ elabora una de las críticas más penetrantes del pensamiento racional cartesiano, señalando que es equivocado separar ser y mundo, sujeto y objeto, res cogitans y res extensa. Propone que el fenómeno primario de la existencia humana es lo que denomina Dasein, es decir "ser-en-el-mundo", es decir no hay un ser que no esté en el mundo, ni un mundo que no lo sea para el ser; ser y mundo se constituyen en simultaneidad y por referencia al otro.

Heidegger explica que la interpretación racionalista del mundo es producto de la práctica filosófica (consciente, reflexiva, racional, deliberativa) de sus mentores, la cual ha sido tomada como modelo general para toda la acción humana. Mientras que alternativamente postula el concepto de transparencia, como la actividad no consciente, no reflexiva, no racional, no deliberativa, que viene a ser la base y condición primaria de la existencia humana. Es decir que ciertas acciones básicas como caminar, manejar el carro, comer en la mesa, etc., lo hacemos en transparencia, es decir que no prestamos atención a cada paso, cada movimiento o combinación de movimientos, tampoco proyectamos por anticipado el movimiento que haremos a continuación, actuamos sin clara conciencia del pavimento que pisamos, de los pedales del carro, del tenedor que tenemos en las manos, nuestra atención está en la conversación que tenemos en ese momento, en las preocupaciones de la agenda que nos espera, etc. Esta ausencia de atención en las acciones transparentes, no implica que se haya suspendido del todo la conciencia y nuestros sentidos, ya que de ser así no nos hubiéramos detenido frente a otro carro o a una luz roja. Es como un flujo de nuestras vidas en sintonía con el mundo que lo rodea.

Heidegger sostiene que sólo emerge la deliberación, la conciencia de lo que estamos ejecutando, cuando este fluir en la transparencia, se ve interrumpido por alguna razón, produciéndose lo que se llama un quiebre. Por ejemplo, si al estar caminando tropezamos, súbitamente observaremos aquel pavimento que antes nos era transparente; si al estar manejando el motor deja de funcionar entonces el carro se nos presenta como un objeto; si al estar comiendo la carne de nuestro tenedor cae en la mesa, dejaremos de prestar atención a la conversación que estamos teniendo en ese momento. 
Estos quiebres suscitan que aquello que antes era transparente emerga ahora en nuestro campo de atención y tomemos conciencia de ello. Sólo entonces nuestra acción se regirá por los patrones de la acción racional, es decir constituímos la relación sujeto-objeto y comenzamos a pensar en cómo restablecer la transparencia perdida. La acción racional resulta ser como un puente que une situaciones de transparencia y surge cuando se produce un quiebre de una acción transparente.

¿Qué resulta ser entonces un quiebre? Involucra también un juicio, de que aquello que acontece, sea lo que sea, no cumple con lo que esperábamos que aconteciera, es decir cuando ocurre una alteración del curso esperado de los acontecimientos. Ello implica que si no tuvieramos la capacidad de hacer juicios, no tendríamos quiebres. Por ejemplo, estamos manejando, llevamos a nuestro perro en el asiento de atrás, y se nos pincha un neumático. Esto representa un quiebre. Cuando manejamos no esperamos que un neumático se nos pinche, resulta en todo caso una remota posibilidad. Mientras ello no suceda los neumáticos no serán transparentes, pero en el caso de ocurrir el pinchazo nos vemos obligados a detener el auto y preocuparnos por el cambio del neumático, lo cual constituye un quiebre para nosotros mas no para el perro que nos acompaña, ya que el mismo no tiene un juicio de lo que ha acontecido, como tampoco lo tiene respecto de los juicios que era esperable que sucediera. En síntesis, la transparencia se quiebra dado los juicios que hacemos sobre lo que acontece, respecto de un trasfondo de juicios sobre lo que es posible y esperado.

Dado que los quiebres habitan en el observador, podemos diseñar la formulación de quiebres, es decir no tenemos que esperar determinados hechos, podemos declarar los quiebres, al menos en lo que respecta al dominio de autoridad de nuestra individualidad. Ello implica la posibilidad de diseñar nuestra vida y futuro. Si la persona tiene autoridad en una organización, puede declarar quiebres respecto de determinados dominios funcionales: "ya no es satisfactorio recibir un 5\% de la tasa de retorno de la inversión", "En vez de ganar nuevos clientes, nuestra preocuopación será la mejora de nuestros servicios y productos", etc. De esta manera, algo que no era un problema en el pasado, lo declaramos como tal en la actualidad, o bien algo que era un problema, deja de serlo a través de una declaración. 
En la medida que estamos planteando que el lenguaje es acción y que nuestras acciones nos hacen ser como somos, podemos concluir que al cambiar nuestra forma de actuar (asumir los compromisos de nuestros actos lingüísticos, por ejemplo), cambiamos nuestra forma de ser. Por lo tanto, somos según como actuamos. Este planteamiento nos remite a pensar la relación persona-acción, no sólo desde el tradicional enfoque agente-acción, sino desde la perspectiva en que las acciones están produciendo a la persona. Así, nuestra identidad depende de las acciones que realicemos.

Figura No.2: Circularidad Ontológica entre Agente y Acción (Dasein)

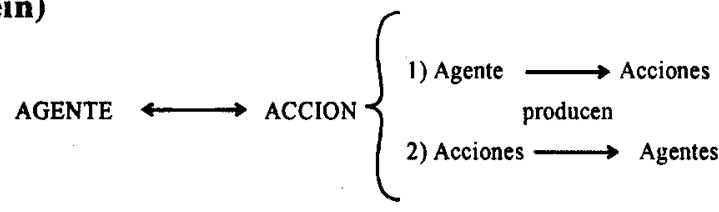

(Fuente: Elaboracion propia)

¿Qué es la acción? Nuevamente con los presupuestos construidos, podemos señalar que la acción es una distinción lingüística. Ya podemos entrever como esta distinción se vuelve crítica de una acción concebida como intencional, a la manera como lo propone Ludwig von Mises (1966): "la acción humana es comportamiento con propósito", es decir una interpretación racional de la acción humana.

En primer lugar, hagamos la distinción de actividad y no de acción cuando estamos describiendo mediante afirmaciones el comportamiento de una persona (levantar el brazo, hablar, ladear la cabeza, caminar). Mientras que la acción humana es una actividad más interpretación, es decir cuando se confiere sentido respecto del comportamiento de esa persona ( $\mathrm{Q}$ Qué es lo que realmente quiere hacer?, ¿Por qué lo hace?, ¿Qué preocupaciones tiene?, etc.). Lo interesante de esta segunda forma de observar el comportamiento es el hecho de que sea el observador quien hace que la acción tenga sentido, por lo que distintos observadores pueden hacer juicios distintos sobre una misma situación. Más aún, un mismo observador, dependiendo de las diversas personas con la que interactúe, puede conferir diferentes interpretaciones de su propio quehacer. Todas las respuestas interpretativas son válidas desde el punto de vista del observador, por que cada una de ellas re-interpreta de manera diferente lo que observa. Sólo al comprender el papel de la interpretación en la 
especificación de la acción, comprendemos el carácter profundamente lingüístico de la acción humana.

Una distinción interesante de la acción es la que nos permite clasificarla como contingente o recurrente. La acción contingente es la acción que se genera cuando no disponemos de una forma establecida de actuar, de tal manera que la acción es contingente a la naturaleza de las circunstancias existentes. Mientras que la acción recurrente proviene de hacer las cosas de determinada manera, para esto las comunidades crean una serie de estructuras de acción que permiten la ejecución recurrente de la acción. También se da el nombre de prácticas sociales a estas recurrencias.

Las prácticas sociales son históricas y varían con el tiempo. Una de sus principales características es que tienden a hacerse transparentes. Es decir la gente las repite sin ponerse a pensar en lo que viene realizando. Las ventajas de este proceso es que permite alcanzar determinados niveles de efectividad, dado que la habilidad para realizarlos nos permite desplazar nuestra atención a otros quehaceres, es decir expande nuestra capacidad de acción. Existen infinidad de prácticas sociales: hablar por teléfono, manejar vehículos en la carretera, pasear por el parque, etc. Sin embargo, también tenemos un aspecto negativo de la rutinización de la acción, y es que al volverse transparentes las prácticas sociales, perdemos nuestra capacidad de observación de las mismas. Este proceso de habituación genera una incapacidad crítica para analizarlas o mejorarlas, perdemos capacidad para aprender e innovar estas acciones recurrentes.

En general, son las organizaciones las que se benefician del poder que tienen las prácticas sociales, por medio de las cuales se reunen muchos individuos para operar de manera transparente y continuada. Una organización con pocas prácticas sociales (pocos sistemas establecidos) gasta una mayor cantidad de tiempo en coordinar y realizar acciones. Por lo que se deduce que hay mucho que ganar en el proceso de diseñar prácticas sociales que permitan una coordinación más fluída de un colectivo. Sin embargo, una excesiva recurrencia hace que la organización se vuelva resistente a los cambios, conservadora, lo cual le hace perder capacidad competitiva. Las tecnologías de "reingeniería", "calidad total", "diseño de procesos" tienen como objetivo rediseñar prácticas habituales de una organización, que por ser transparentes tienden a ocultarse y dejan de ser objeto de evaluación y mejora. 
Otra forma en que aparece la relación entre acción y lenguaje es a través del hecho que podemos proceder a una reconstrucción linguística de las prácticas sociales. Al hacerlo tratamos cada práctica social como si se tratara de un juego con determinados objetivos y reglas que lo especifican. Fue Wittgenstein quien propuso esta idea de examinar el lenguaje como juegos y que tomamos para utilizarla para una reconstrucción lingüística de determinadas prácticas sociales. Los juegos son útiles para ampliar nuestra comprensión de las prácticas sociales, a la manera de los "manuales de instrucción" que utilizan el lenguaje para introducirnos en determinadas prácticas. Nos guían, paso a paso, a través del lenguaje, en el aprendizaje de una práctica social como si fuese un juego.

Así, cuando jugamos un juego podemos identificar clases de reglas, las cuales fueron constituídas por declaraciones, y que las podemos agrupar en cinco categorías:

a) Declaraciones constitutivas, donde se señala lo que los jugadores persiguen en su actuación, es decir definen el objetivo o propósito del juego, por ejemplo en el caso del fútbol: ganar metiendo más goles que el adversario.

b) Declaraciones de existencia, que explican las condiciones necesarias para ganar el juego, así como el ámbito espacial y temporal dentro de la cual se lleva la acción. Para el mismo ejemplo anterior: el tamaño de la cancha y de los arcos, dos tiempos de 45 minutos, dos equipos de 11 jugadores, la presencia de un arquero en cada equipo, una pelota, un árbitro, lo que es un gol, etc.

c) Declaraciones de acción: que viene a ser las leyes de acción del juego, es decir lo que les está permitido, prohibido y lo que es obligatorio para cada jugador. Por ejemplo, no se puede tocar la pelota con la mano excepto para el arquero dentro de su área, no se puede golpear a un jugador, hay que sacar el corner o el lateral de una determinada manera. Estas declaraciones suelen acompañarse de sanciones.

d) Declaraciones para la resolución de conflictos: que tratan de resolver los vacios cuando hay una situación no prevista por las anteriores reglas, y que exigen una instancia superior de autoridad que el mismo árbitro. En el caso del fútbol: un Tribunal de Disciplina, Asociación Nacional de fútbol, la FIFA, etc.

e) Reglas Estratégicas: las cuales no constituyen declaraciones y que provienen del aprendizaje de experiencias anteriores a lo largo de la historia de la práctica social y que son usadas electivamente 
para mejorar la eficiencia de los jugadores. Así, la regla de alineación de jugadores en la cancha: 4-3-3 o 4-2-4 o 4-4-2.

$\mathrm{Al}$ hacer esta reconstrucción lingüística de las prácticas sociales se abre un nuevo dominio de acción: la innovación y el diseño. Una vez reconstruída una práctica social existente, podemos jugar libremente con cada uno de sus componentes y modificar algunos de ellos. Cuando hay cambios en alguna de ellas estamos frente a una práctica social diferente (en el caso de disminuir las dimensiones de la cancha, estamos frente a otro juego que se denomina fúbol rápido o fulbito), y encontrar formas más efectivas de realizar una práctica social.

Diseñar nuevas formas de prácticas sociales implica establecer con claridad cúal es el objetivo del juego, sus diversas declaraciones, para luego juzgar que podemos corregir para aumentar su efectividad de acuerdo a nuestros intereses. Además, cada vez que nos enfrentamos a un quiebre recurrente, si no se ha establecido una práctica social que nos regrese al estado de transparencia, podemos diseñar una práctica social ad-hoc.

\section{Hacia una teoría lingüística de la Organización}

Intentemos ahora una perspectiva teórica que asuma a las organizaciones como entidades socio-linguísticas, aplicando las ideas desarrolladas en los acápites anteriores sobretodo en cuanto al papel generativo del lenguaje. Las organizaciones son fenómenos lingüísticos en tanto son unidades construidas a partir de diversos actos lingüísticos concatenados que conocemos como CONVERSACIONES, las cuales están basadas en la capacidad de los seres humanos para efectuar compromisos mutuos cuando se comunican entre sí.

Una organización no viene a ser tanto su nombre, el espacio público donde se ubica o el servicio o producto por el cual se constituye. Todos ellos pueden cambiar y la organización seguirá existiendo. Una organización es sobre todo una red estable y estructurada de conversaciones, y como tal genera una identidad que trasciende a sus miembros integrantes. La organización, en tanto comprometida con los actos lingüísticos que emite, es una unidad de acción; es decir es socialmente responsable frente a sus interlocutores. 
Sin lenguaje no podríamos construir organizaciones. Es mediante las conversaciones que las organizaciones definen sus límites y por consecuencia a quienes son sus miembros y a su entorno, al cual hace ofertas y acepta peticiones, que resultan en promesas que la actividad interna de la organización se encarga de satisfacer. Podemos observar que es través de lo que ya conocemos como DECLARACIONES, que se define quienes son los miembros de la organización, ya que alguién investido de autoridad al interior de la organización, acepta la petición de ingreso y formula la declaración. Estos límites no son permanentes, ya que por otras declaraciones se separa a un miembro de la organización, ya sea por que se la despide o por que se acepta su petición de retiro voluntario.

La estructura interna de una organización está construída por una red de promesas mutuas. Cada miembro está ligado por compromisos específicos y es responsable de cumplir con algunas condiciones de satisfacción determinadas. Estas promesas configuradas por medio de conversaciones (peticiones u ofertas y declaraciones de aceptación) se expanden a lo largo de la organización en múltiples dominios; por ejemplo: producción, ventas, markentig, servicio al consumidor, administración, gerencia general, en el caso de organización empresarial. La especificidad y agrupamiento de actos lingüísticos para el cumplimento de promesas van configurando la estructura interna de una organización. Esta división de los actos lingüísticos como sustrato de una división social del trabajo, tiene un marco linguiístico de coordinación general de toda la organización, para permitir que se cumplan con las promesas que la organización tiene con el entorno.

Una organización es más que una red de individuos autonómos y con intereses propios, ligados por una declaración de pertenencia al mismo colectivo. Otra dimensión a considerar es que estos individuos desempeñan sus acciones sobre la base de un trasfondo compartido, la cual constituye una gran ventaja económica en lo que significa "costos de transacción", ya que cada intercambio efectuado se hace sobre la base de que la gente tiene la consistencia y la competencia para cumplir la promesa realizada. Este trasfondo compartido implica asimismo una generación de condiciones sinérgicas que permite una coordinación óptima, o en todo caso mejor que si una sola persona realizase la actividad (que implicaría pérdida de calidad y cantidad por la ausencia de la especialización) o que distintas organizaciones tuviesen que llevar a cabo una misma tarea (que incrementa los costos de tiempo en homogeneizar criterios y especialidades). 
Este transfondo compartido se conoce mejor como la "cultura" de una organización, la cual permite el desarrollo de prácticas sociales propias de la organización, de estándares sociales comunes desde los cuales los individuos emiten juicios, de formas compartidas de actuar y hacer frente a las circunstancias para producir resultados. Estas prácticas sociales van generando una transparencia organizacional, a la manera de conjunto de hábitos repetitivos y cuasi-automáticos para los individuos que están inmersos en ellos.

Otro aspecto importante, en el que se reconoce como el lenguaje actúa como un articulador y generador de una organización, es la construcción de una perspectiva compartida respecto del futuro de la organización, es lo que comúnmente se conoce como MISIÓN y VISIÓN, que forma parte también de una cultura organizacional. Esta visión y misión ayudan a fortalecer las condiciones sinérgicas de la organización, ya que genera compromisos colectivos para alcanzar los intereses mutuos que se generan a partir de las conversaciones al interior de la organización. Este futuro compartido que se crea colectivamente es la base consensual necesaria para dar viabilidad y continuidad a la organización.

De este tipo de enfoque lingüístico se desprende una propuesta diferente para analizar empíricamente a las organizaciones. El planteamiento consiste en que toda la dinámica organizacional transcurre a partir de actos lingüísticos. Por lo tanto, si queremos comprender a una empresa debemos examinar las conversaciones que la constituyeron en el origen y las que la constituyen en la actualidad. Así, tanto sus fortalezas como debilidades nos remitirán a las fortalezas o debilidades en el cumplimiento de sus diversos actos linguïsticos (Ver Cuadro No. 1, supra pag. 15). Asimismo, es posible analizar cúales de las transparencias existentes (hábitos) contribuyen positivamente con los objetivos de la organización, y cuáles son negativas en el sentido que constituyen "cegueras" al interior de la organización, ya que siendo obstáculos para los intereses del actor colectivo, no son percibidas como tales sino como procedimientos "naturales" o "normales" dentro de la organización.

Un analista organizacional desde el enfoque lingüístico cumple con desempeñar un rol de observador privilegiado, ya que al no compartir las transparencias ni la cultura organizacional, puede detectar y revelar puntos ciegos que constituyen obstáculos en el desempeño organizacional, así también puede intervenir en los procesos conversacionales y resaltar las insuficiencias en la falta de 
compromisos de los actos lingüísticos emitidos por los miembros y en otras fallas de la comunicación (por ejemplo, cuando las condiciones de satisfacción respecto de una promesa han sido entendidas de manera diferente por las personas vinculadas en la constitución de una promesa), las cuales interfieren en el proceso de la coordinación de la acción generando ineficiencia e ineficacia en la dinámica organizacional.

Incluso, esta teoría propone que cualquier problema, sea o no comunicativo, puede ser examinado desde la perspectiva de sus conversaciones subyacentes. Ello no implica que no se reconozca dimensiones no conversacionales, como por ejemplo problemas técnicos, financieros, logísticos, etc. Empero, aún estos problemas pueden ser abordados a través de los actos lingüísticos, ya sea de aquellos que se llevaron a cabo sobre una base no consensual de entendimiento, o sobre el incumplimiento de los compromisos a que dieron lugar dichos actos lingüísticos. Nos referimos más precisamente a conversaciones que no establecieron las afirmaciones adecuadas acerca de la descripción y uso de un equipo técnico, las declaraciones que fueron incumplidas en el pago de una deuda que originaron el reclamo del banco, los juicios no fundados sobre los cuales se programó las medidas de aprovisionamiento para un periodo determinado. La atención sobre las competencias comunicativas de una organización nos lleva a entender que todo lo que ocurre al interior de la misma puede ser examinado desde la deconstrucción de sus conversaciones.

La historia de la organización se puede analizar desde sus origenes mediante las declaraciones constitutivas (acta de fundación, registro inaugural); asimismo por las declaraciones posteriores que asientan las reglas de juego que configuran la dinámica organizacional, así como por declaraciones de acción que asignan responsabilidades, señalan metas y estrategias, celebran contratos, establecen alianzas con otras organizaciones.

Un claro ejemplo de la naturaleza conversacional de las organizaciones la tenemos en el nivel directivo de las organizaciones. Si estudiamos el rol que los gerentes o ejecutivos hacen dentro de una empresa, nos daremos cuenta que su trabajo consiste casi exclusivamente en "estar" en conversaciones. Como señala Mintzberg (1983: 55): "El trabajo del directivo consiste en contactos verbales y escritos, y sus principales instrumentos son: la documentación de 
oficina, el teléfono, las reuniones imprevistas, las reuniones concertadas y las giras".

Figura 3: Distribución del Número de Actividades del Directivo

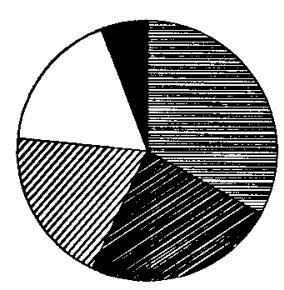

\begin{tabular}{|l|}
\hline Trabajo de Oficina $33 \%$ \\
Llamadas Telefónicas $24 \%$ \\
Reuniones Imprevistas $24 \%$ \\
Reuniones Programadas \\
$19 \%$ \\
Giras de Inspección $5 \%$
\end{tabular}

Figura 4: Distribución de horas de las actividades del Directivo

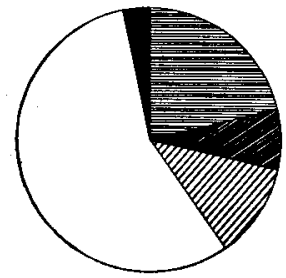

- Trabajo de Oficina $22 \%$

Llamadas Telefónicas $6 \%$

$\mathbb{Z}$ Reuniones imprevistas $10 \%$

$\square$ Reuniones Programadas $59 \%$

Giras de Inspección $3 \%$

(Fuente: Mintzberg; 1983)

Como muestran los gráficos anteriores extraídos de Mintzberg (1983; 55-84), que elaboró a partir de la observación empírica del trabajo de altos ejecutivos, el directivo ocupa la mayor parte de su tiempo en contactos verbales (75\%). Por otro lado, se señala que los propósitos más recurrentes en tales contactos verbales son: recepción de información, revisión, formulación de estrategia, solicitudes de acción, participación de ceremonias y negociación.

Figura 5: Distribución de horas de contacto verbal según su objeto

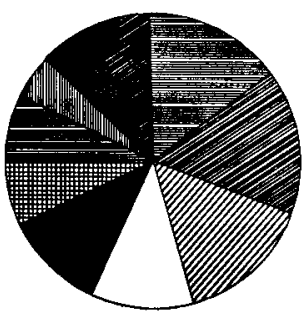

(Fuente: Mintzberg; 1983)
目 Recepción de información $15 \%$

Revisión de documentos $15 \%$

Elaboración de estrategias $13 \%$

Solicitudes de acción $12 \%$

Participación de ceremonias $12 \%$

Feuniones de Negociación $8 \%$ 
Como consecuencia de sus observaciones, Mintzberg propone las siguientes características específicas del trabajo del alto directivo:

a) El ejecutivo realiza un enorme volumen de trabajo, dispone de poco tiempo libre y pocos descansos.

b) Las actividades del directivo son breves, variadas y fragmentadas.

c) El directivo prefiere las actividades más activas de su trabajo, es decir las que sean inmediatas, concretas, definidas y no rutinarias.

d) El trabajo del directivo consiste principalmente en establecer contactos verbales y escritos, aunque en preferencia los primeros.

e) El ejecutivo actúa como enlace entre la organización y el entorno, dedicando entre un $30 \%$ y un $50 \%$ de su tiempo en establecer estos contactos.

f) El ejecutivo dedica entre un $30 \%$ y un $50 \%$ de su tiempo en establecer contactos con sus subordinados para dar instrucciones, recibir información y hacer planes.

Las conclusiones de Mintzberg confirman la importancia generativa que el lenguaje tiene en la dinámica de las organizaciones, permitiendo no sólo la existencia de la organización sino además su continuidad y éxito (o fracaso). Además, nos resalta la necesaria transparencia (hábitos recurrentes) que han de existir en la organización para que permita el trabajo creativo, contingente y planificador que el ejecutivo tiene hacia adentro y fuera de la organización.

\section{Algunas Conclusiones a manera de Síntesis}

Hemos hecho un largo recorrido para presentar a las organizaciones como redes recurrentes de compromisos humanos que pasan por el lenguaje; a su vez hemos analizado el lenguaje como algo más que palabras, como un sistema que constituye nuestro mundo como relaciones y como actores, los cuales se integran a través de actos lingüísticos.

A partir de este enfoque, podemos señalar que la tesis central del mismo es entender a las organizaciones como redes de conversaciones que generan compromisos lingüísticos. Esta tesis nos conduce a una concepción distinta de la concepción tradicional de realidad y verdad, entendida ésta como una manifestación pasiva de aquella. Ahora nos 
proyectamos a una verdad interpretativa, que surge de las prácticas comunicativas de comunidades humanas y que no está por encima de ellas.

Los objetos de la realidad se constituyen al conversar, sobretodo los objetos de carácter social (conceptos, procesos, temporalidades, relaciones, etc.). Somos cuerpos que se asoman a un espacio histórico social y que al encontrarse con el lenguaje, nos constituímos en actores. Una constitución que se funda en el mutuo reconocimiento social en base a la red de compromisos que se generan en la conversación. Estos compromisos dan cuenta de los deseos e intereses de los actores en base a un sistema de coorperación que activa una red acciones colectivas, uno de estos sistemas son las organizaciones.

Las organizaciones son un fenómeno producido por el lenguaje, inicialmente por aquellas declaraciones constitutivas por la que se comprometen determinados individuos a coordinar sus acciones por medio de una división social del trabajo, la cual da lugar a nuevas declaraciones de existencia y acción que específica las condiciones en las que la coordinación se ha de efectuar. Las declaraciones proporcionan la estabilización de los roles, esto es, aceptaciones normales de peticiones recurrentes en ámbitos recurrentes. Por tanto, si lo que se postula y comprueba es que la principal actividad de la gente en las organizaciones es conversar; necesitamos entonces descubrir qué clases de actos lingüísticos constituyen una conducta organizacional. Por ejemplo, un acercamiento analítico de las declaraciones nos permitirá identificar los ámbitos relevantes de la organización.

Una organización no sólo se mantiene unida mediante patrones de conversación recurrentes, sino también por un trasfondo compartido, que solemos denominar como "cultura organizacional" o conjunto de valores o creencias. Este trasfondo está constituído por declaraciones asentadas y asumidas por todos los miembros que la comparten. Este trasfondo permite a los miembros (y sólo a ellos) dar sentido a las conversaciones nuevas que producen la acción colectiva organizacional, a las conversaciones sobre estas conversaciones dirigidas a evaluar esas mismas acciones, y finalmente a las conversaciones que producen nuevas posibilidades y oportunidades al interior de la organización.

Este nuevo concepto de organización permite entender la noción de estructura organizacional a través del concepto de transparencia, es decir de actos lingüísticos recurrentes que se constituyen en hábitos 
que facilitan la coordinación de actividades. Estructuras que constituyen, muchas veces, serios obstáculos para mejorar la eficiencia de esas mismas actividades y que son fuente de problemas latentes que afectan la dinámica organizacional.

\section{Bibliografía}

Austin, J.L. (1982). Como hacer las cosas con palabras. Barcelona: Paidós.

Echevarría, Rafael (1993). El búho de Minerva. Santiago de Chile: Dolmen.

Echevarría, Rafael (1994). Ontología del Lenguaje. Santiago de Chile: Dolmen.

Flores, Fernando (1997). Creando organizaciones para el futuro. Santiago de Chile: Dolmen.

Heidegger, Martin (1982). On the way to language. San Francisco: Harper \& Row.

Heidegger, Martin (1985). El Habla. Espacios, II, 6.

Maturana, Humberto (1990). Biología de la cognición y epistemología. Temuco, Chile: Ediciones de la Universidad de la Frontera.

Maturana, Humberto (1992). El sentido de lo humano. Santiago de Chile: Hachette.

Mayr, Ernest (1963). Animal Species and Evolution. Cambridge,

Mass.: Bellknap Press of Harvard University Press.

Mintzberg, Henry (1983). The Nature of Managerial Work.

Englewood Cliffs, N.J.: Prentice-Hall

Nietzsche, Friedrich (1986). Genealogía de la Moral. Madrid:

Alianza.

Pears, David (1971). Wittgenstein. Londres: Fontana-Collins.

Searle, John (Ed.) (1979). The Philosophy of Language, Oxford:

Oxford University Press.

Searle, John (1980). Actos de Habla. Madrid: Cátedra.

Wittgenstein, Ludwig (1968). Philosophical Investigations. New

York: Macmillan,

Wittgenstein, Ludwig (1989). Conferencia sobre ética. Barcelona:

Ediciones Paidós, 